\title{
CONSTRUCTING BANASCHEWSKI COMPACTIFICATION WITHOUT DEDEKIND COMPLETENESS AXIOM
}

\author{
S. K. ACHARYYA, K. C. CHATTOPADHYAY, and PARTHA PRATIM GHOSH
}

Received 14 May 2003

\begin{abstract}
The main aim of this paper is to provide a construction of the Banaschewski compactification of a zero-dimensional Hausdorff topological space as a structure space of a ring of ordered field-valued continuous functions on the space, and thereby exhibit the independence of the construction from any completeness axiom for an ordered field. In the process of describing this construction we have generalized the classical versions of M. H. Stone's theorem, the Banach-Stone theorem, and the Gelfand-Kolmogoroff theorem. The paper is concluded with a conjecture of a split in the class of all zero-dimensional but not strongly zero-dimensional Hausdorff topological spaces into three classes that are labeled by inequalities between three compactifications of $X$, namely, the Stone-Čech compactification $\beta X$, the Banaschewski compactification $\beta_{0} X$, and the structure space $2 \mathfrak{n}_{X, F}$ of the lattice-ordered commutative ring $\mathfrak{C}(X, F)$ of all continuous functions on $X$ taking values in the ordered field $F$, equipped with its order topology. Some open problems are also stated.
\end{abstract}

2000 Mathematics Subject Classification: 54D80, 54D35, 12J15, 06F25.

1. Introduction. The main thrust in the area of rings of real-valued continuous functions defined over a topological space $X$ was provided by the three historical papers due to Stone [29], Gelfand and Kolmogoroff [14], and Hewitt [18]. Stone initially assumed a metric structure on the underlying space and his study was confined to bounded real-valued continuous functions only, that is, $\mathfrak{C}^{*}(X, \mathbb{R})$ in the notation used by Hewitt; Gelfand and Kolmogoroff dropped the metric structure for the first time from the underlying space and replaced it by the more general topological space, thereby paving the way for a study of $\mathfrak{C}(X, \mathbb{R})$. Finally Hewitt introduced his almost omnipresent $\mathbb{Q}$-spaces, presently called the realcompact topological spaces, providing the final shape to the research work for the subsequent years. All this culminated in the classic textbook [15].

Of the many areas of research that were influenced by this textbook, some of those relevant to the main theme of this paper are the following.

1.1. Other topological algebras as range. (1) Paper [19] considers the rings of continuous functions with values in a topological ring.

(2) Paper [24] considers the rings of continuous functions with values in the ring $\mathbb{Z}$.

(3) Paper [3] considers the rings of continuous functions with values in a topological field whose topology is derived from a complete ultrametric.

(4) In [37], the author considers the rings of continuous functions with values in a topological skew field.

(5) In [39], the author provides a historical sketch and a wide-ranging survey of the problem of associating with every topological space $X$ an algebraic structure $A(X)$, in 
such a way that when two spaces $X$ and $Y$ are homeomorphic the algebraic structures $A(X)$ and $A(Y)$ are isomorphic. The author then describes how this gives rise to the descriptions of topological spaces that are determined by a kind of algebraic structures. In [40], the algebraic aspects of the theory of rings of continuous functions are nicely surveyed.

(6) Papers [1, 2] consider the hemiring of continuous functions with values in the hemiring $\mathbb{R}_{\geq 0}$ of nonnegative reals.

1.2. The problem of determining topological spaces. (1) The first determination of a class of topological space by an algebraic structure was in the papers [30, 31] which solved the problem of determining compact Hausdorff spaces using the ring of bounded real-valued continuous functions. This was followed by [18], with the problem of determining the realcompact spaces by the rings of real-valued continuous functions. Generalizations of Hewitt's results appear in [27, 28].

(2) In $[8,20]$, it was shown that the multiplicative semigroup structure on the semigroup of real-valued continuous functions on a topological space determines the class of completely regular spaces, while [33] generalizes the range to a topological nonassociative division ring which is either locally compact or totally disconnected, and proves a similar result for a class of $T_{1}$ spaces satisfying quite natural regularity conditions.

(3) Paper [16] shows that the rings of real-valued continuous functions on two completely regular topological spaces are isomorphic if and only if the two are isomorphic as lattices or as multiplicative semigroups.

1.3. Ideal structure. (1) Apart from the study of the structure spaces in the pioneering papers of Hewitt and Stone, an attempt to study the ideal structure of the rings of continuous functions with values in a non-Archimedean ordered field was done in [11] where the residue class fields are investigated.

(2) In $[34,35,38]$, the projective and injective ideals of the rings of continuous functions are studied.

(3) In [41, 42, 44], the maximal and prime spectra of the rings of continuous functions taking values in a Hausdorff topological field are investigated.

(4) The connections with sheaf theory are discussed in [32, 43, 45].

1.4. $f$-rings. The generalization of the rings of continuous functions into $f$-rings has provided great impetus towards obtaining properties for these rings in recent years. A good survey of the investigations in this area can be obtained in [17] and a brief introduction to this area can be found in [5]. It is only once that the notions of $f$-rings will be referred to in this paper, for purposes which the papers $[4,6,7]$ suffice.

The present paper deals with the following question of how much of the present theory of rings of continuous functions depends on the first-order properties of the ordered field. Thus we want to investigate the results of [15] that can be extended to our situation, where the range field $\mathbb{R}$ is replaced by a linearly ordered field $F$ equipped with its order topology. The investigation shows that while many results can be extended, some can only be partially extended-in the sense that one requires some more regularity properties on the order of the field, like Dedekind completeness or countable cofinality, to hold good for the extension to be true, and some possibly would yield 
completely new results - the split in Section 4, when the ordered field is not that of the reals.

Throughout this paper the term order refers to a linear order and an ordered field is always equipped with its order topology; all topological spaces that appear here are at least Hausdorff. The paper is organized as follows.

In Section 2, the rings of continuous functions, bounded functions, and functions with compact codomain from a topological space to an ordered field are introduced and some basic properties of these function rings are established. Theorem 2.5 characterizes the real field $\mathbb{R}$ and Theorem 2.7 characterizes ordered fields with countable cofinality character, and seems to be new. Furthermore, the topological property of complete $F$-regularity ( $F$ is an ordered field) is defined and it is shown that this is the right kind of spaces that one can study using an ordered field $F$; see Theorem 2.15. Indeed this is exactly the generalization of M. H. Stone's theorem; see [15, Theorem 3.9, page 41]. Also, various obvious characterizations of $F$-regular topological spaces are provided in Theorems 2.10 and 2.14.

In Section 3, we describe the structure space of the function rings described in Section 2. Most of the results in this section seem to be new. Generalizations of the classical Banach-Stone theorem (Theorem 3.3) and the Gelfand-Kolmogoroff theorem (Theorem 3.5) are obtained. The main results of the paper appear in this section-Theorems 3.10 and 3.12, which provide a plethora of ways to construct the Banaschewski compactification $\beta_{0} X$ of a zero-dimensional Hausdorff topological space. Theorem 3.11 is stated as a separate result; it is an immediate consequence of the observations made before and helps in conjecturing the split in Section 4.

In Section 4, we finally summarize the results of Section 3 which culminates in the description of the split. There are some questions that remain unsolved and are proposed at the end.

2. Preliminaries. Throughout this section, $X$ is a topological space and $F$ is an ordered field. We start with various definitions.

\section{DEFINITION 2.1.}

$$
\begin{aligned}
\mathfrak{C}(X, F) & =\left\{f \in F^{X}: f \text { is continuous on } X\right\}, \\
\mathfrak{B}(X, F) & =\{f \in \mathfrak{C}(X, F):(\exists t \in F)(\forall x \in X)(-t \leq f(x) \leq t)\}, \\
\mathfrak{C}^{*}(X, F) & =\left\{f \in \mathfrak{C}(X, F): \mathrm{cl}_{F}(f(X)) \text { is compact }\right\}, \\
\mathscr{L}_{X, F}(f) & =\{x \in X: f(x)=0\}, \text { for } f \in \mathfrak{C}(X, F), \\
\mathfrak{Z}(X, F) & =\left\{\mathscr{L}_{X, F}(f): f \in \mathfrak{C}(X, F)\right\} .
\end{aligned}
$$

A subset $A \subseteq X$ is said to be a zero set in $X$ with respect to $F$ if and only if there exists an $f \in \mathfrak{C}(X, F)$ such that $A=\mathscr{E}_{X, F}(f)$; the complement of a zero set in $X$ with respect to $F$ is called a cozero set in $X$ with respect to $F$. The cozero set in $X$ with respect to $F$ of $f \in \mathfrak{C}(X, F)$ will be denoted by $\operatorname{coz}_{X, F}(f)$.

One can easily see that $F \subseteq \mathfrak{C}^{*}(X, F) \subseteq \mathfrak{2}(X, F) \subseteq \mathfrak{C}(X, F)$, if one identifies the member $t \in F$ with the constant function $X \stackrel{\mathbf{t}}{\rightarrow} F$, where $\mathbf{t}: x \mapsto t$. Also, if one assigns the operations of addition, multiplication and maximum and minimum pointwise on 
the functions, then one easily gets $\mathfrak{C}(X, F)$ to be a lattice-ordered commutative ring with unity, and gets $\mathfrak{B}(X, F)$ and $\mathfrak{C}^{*}(X, F)$ to be lattice-ordered commutative subrings of $\mathfrak{C}(X, F)$.

It is easy to see that if $X$ has at least two points, then no lattice-ordered subring of $\mathfrak{C}^{*}(X, F)$ containing $F$, other than $F$ itself, can be a field, so that the rings in question always have divisors of zero. The following results are useful in the sequel.

THEOREM 2.2. (1) Any topological field is either connected or totally disconnected (see [46, page 213]).

(2) An ordered field either is isomorphic to $\mathbb{R}$, the field of real numbers, or is zero dimensional.

Proof. (1) Let $K$ be a topological field and let $W$ be the component of 0 . Let, for any $x \in K$ and any $H \subseteq K, x H=\{x h: h \in H\}$.

If $W=\{0\}$, then the field is totally disconnected; if not, let $x \in W$ and $x \neq 0$. Then $x^{-1} W$ is a connected subset of $K$ that contains 1 , and hence $K=\bigcup_{y \in K} y x^{-1} W$. However, since each of the sets $y x^{-1} W$ are connected and $0 \in y x^{-1} W$, it follows that the set $\bigcup_{y \in K} y x^{-1} W$ is connected and hence $K$ is connected.

(2) From (1), an ordered field is either connected or else totally disconnected. Since an ordered set is connected if and only if it is Dedekind complete [15, Problem 30, page 52], it follows that if $F$ as an ordered field is connected, then it is isomorphic to $\mathbb{R}$.

Let $F$ be an ordered field that is totally disconnected and let $F^{*}$ be the Dedekind completion of $F$. Then it is clear that $\left\{\left(a^{*}, b^{*}\right) \cap F: a^{*}, b^{*} \in F^{*}\right\}$ is a clopen base for the topology of $F$. Hence $F$ is zero dimensional.

COROLLARY 2.3. Any ordered field, nonisomorphic to $\mathbb{R}$, is neither locally connected nor locally compact in its order topology.

It follows from Theorem 2.2(2) that $F \neq \mathbb{R}$ implies that $\mathfrak{C}^{*}(X, F)$ contains a nonconstant function if and only if $X$ is disconnected. The case for $F=\mathbb{R}$ is settled in [15] as follows: $\mathfrak{C}^{*}(X, \mathbb{R})$ possesses a nonconstant function if and only if $\mathfrak{C}(X, \mathbb{R})$ possesses a nonconstant function, and because every Tychonoff space with at least two points always has a nonconstant continuous function. Hence $\mathfrak{C}(X, \mathbb{R})$ has a nonconstant continuous function when $X$ is a Tychonoff space with at least two points.

The following lemma shows that $\mathfrak{C}(X, F)$ and $\mathfrak{2}(X, F)$ determine the same family of zero sets in $X$ with respect to $F$.

LEMMA 2.4. Given any $f \in \mathfrak{C}(X, F)$, there exists a positive unit $u \in \mathfrak{C}(X, F)$ such that $u f=(-1 \vee f) \wedge \mathbf{1}$.

If, further, $f \in \mathfrak{2} 3(X, F)$, then $u$ can be chosen to be in $23(X, F)$ too.

Proof. For any $f \in \mathfrak{C}(X, F)$, define

$$
u(x)= \begin{cases}\frac{1}{|f(x)|} & \text { if }|f(x)| \geq 1 \\ 1 & \text { otherwise. }\end{cases}
$$

Surely $u$ is continuous and is a candidate required for the proof of the lemma. 
Hence if one of the two rings $\mathfrak{C}(X, F)$ and $\mathfrak{b}(X, F)$ possesses a nonconstant function, the other also does.

The following shows the equality of $\mathfrak{2}(X, F)$ and $\mathfrak{C}^{*}(X, F)$ to be a characteristic property of $\mathbb{R}$.

THEOREM 2.5. For an ordered field $F, F=\mathbb{R}$ if and only if, for any topological space $X, \mathfrak{C}^{*}(X, F)=\mathfrak{2}(X, F)$.

Proof. If $F=\mathbb{R}$, then the conclusion is trivially true. If $F \neq \mathbb{R}$, then the function $F \stackrel{f}{\rightarrow} F$, defined by $f=\min \{g, 1\}$, where

$$
g(x)= \begin{cases}-x & \text { if } x \leq 0 \\ 0 & \text { if } 0 \leq x \leq 1 \\ x-1 & \text { if } x \geq 1\end{cases}
$$

is a member of $\mathfrak{2}(X, F)$, and since by Corollary $2.3[0,1]$ is not compact, it follows that $f$ is not a member of $\mathfrak{C}^{*}(X, F)$.

REMARK 2.6. It is known that the Heine-Borel property for an ordered field is a characteristic property for $\mathbb{R}$. Thus, Theorem 2.5 is a latent exhibition of this equivalence.

As usual the zero sets in $X$ with respect to $F$ are closed subsets of $X$. However, we have the following.

THEOREM 2.7. Let $F$ be any ordered field with cofinality character $\omega_{\alpha}$. Then, the zero sets in $X$ with respect to $F$ are intersections of an $\omega_{\alpha}$ sequence of cozero sets in $X$ with respect to $F$.

In particular, for any topological space $X$, the zero sets in $X$ with respect to $F$ are $G_{\delta}$-subsets of $X$ if and only if $\alpha=0$.

Proof. For any $f \in \mathfrak{C}(X, F), \mathscr{L}_{X, F}(f)=\bigcap_{r \in T}\left\{x \in X:|f(x)|<r^{-1}\right\}$, where each of the sets on the right-hand side are clearly cozero sets in $X$ with respect to $F$ and $T \subseteq F_{>0}=\{x \in F: x>0\}$ is cofinal in $F$.

The "if" part of the final statement follows from the first; for the "only if" part, if $\alpha>0$, then $f(x)=|x|$ is a member of $\mathfrak{C}(X, F), \mathscr{E}_{X, F}(f)=\{0\}$, and $\{0\}$ is not a $G_{\delta}$-set.

However, the following example shows that countable cofinality of an ordered field does not, in general, imply it to be Archimedean, and thus by the above proposition $G_{\delta}$-ness of the zero sets may also occur for many other ordered fields other than $\mathbb{R}$ or its subfields.

EXAMPLE 2.8. Consider the real field $\mathbb{R}$ and consider the polynomial ring $\mathbb{R}[x]$. For $f=a_{0}+a_{1} x+a_{2} x^{2}+\cdots+a_{n} x^{n} \in \mathbb{R}[x]$, define $f>0$ if and only if $a_{n}>0$. Clearly this definition yields a positive cone, so that with the order $f \leq g$ if and only if $g-f>\mathbf{0}$ or $g=f$ it is easy to see that $\mathbb{R}[x]$ is an ordered ring. 
Now consider the field $\mathbb{R}(x)$ of rational polynomials, $f / g, f, g \in \mathbb{R}[x]$, and $g>\mathbf{0}$, and extend the order on $\mathbb{R}[x]$ as follows: for $f, f^{\prime}, g, g^{\prime} \in \mathbb{R}[x]$ with $g, g^{\prime}>\mathbf{0}$, define $f / g \leq f^{\prime} / g^{\prime}$ if and only if $f g^{\prime} \leq f^{\prime} g$ in $\mathbb{R}[x]$. It is easy to see that this makes $\mathbb{R}(x)$ an ordered field.

However, for any natural number $n \in \mathbb{N}, x>\mathbf{n}$ and thus one has that the polynomial $x$ is infinitely larger than every constant polynomial $\mathbf{n}$. Hence $\mathbb{R}(x)$ is not an Archimedean ordered field. But for any member $f / g \in \mathbb{R}(x)$, there exists a natural number $n \in \mathbb{N}$, namely, $n=\operatorname{deg}(f)-\operatorname{deg}(g)+1$, such that $f / g<x^{n}$. Thus the set $\left\{\mathbf{1}, x, x^{2}, \ldots\right\}$ is cofinal in $\mathbb{R}(x)$, showing that the cofinality character of $\mathbb{R}(x)$ is $\omega_{0}$.

DEFINITION 2.9. (1) $A, B \subseteq X$ are said to be completely $F$-separated if and only if there exists an $f \in \mathfrak{C}(X, F)$ such that $f(x)=0$ on $A$ and $f(x)=1$ on $B$.

(2) $X$ is said to be completely $F$-regular if and only if, for every closed subset $A$ of $X$ and every $x \in X \backslash A$, the sets $\{x\}$ and $A$ are completely $F$-separated.

This is the clear analog of the Tychonoff property when $F=\mathbb{R}$. We first provide some equivalent descriptions of this property, in general.

THEOREM 2.10. For any topological space $X$ the following are equivalent:

(1) $X$ is completely F-regular;

(2) $3(X, F)$ is the base for the closed subsets of $X$;

(3) $X$ has the weak topology induced by $\mathfrak{C}(X, F)$;

(4) $\mathfrak{2}(X, F)$ separates points and closed subsets of $X$;

(5) $\mathfrak{C}^{*}(X, F)$ separates points and closed subsets of $X$.

Proof. $\quad(1) \Rightarrow(2)$. Immediate from Definition 2.9(2).

(2) $\Rightarrow(3)$. Let $V \subseteq X$ be an open subset of $X$ and $x \in V$. Then $G=X \backslash V$ is closed and $x \notin G$, so that from the hypothesis there exists an $f \in \mathfrak{C}(X, F)$ such that $G \subseteq \mathscr{L}_{X, F}(f)$ and $f(x) \neq 0$. Hence $x \in \operatorname{coz}_{X, F}(f) \subseteq V$ and thus the class of cozero sets in $X$ with respect to $F$ yields a base for the topology for $X$. But the equations

$$
\begin{gathered}
f^{\leftarrow}((-\infty, a))=\operatorname{coz}_{X, F}((f \wedge \mathbf{a})-\mathbf{a}), \\
f^{\leftarrow}((a, \infty))=(-f)^{\leftarrow}((-\infty,-a)), \\
f^{\leftarrow}((a, b))=f^{\leftarrow}((-\infty, b)) \cap f^{\leftarrow}((a, \infty))
\end{gathered}
$$

show that the family of cozero sets in $X$ with respect to $F$ is precisely the family of all finite intersections of inverse images of open intervals under maps in $\mathfrak{C}(X, F)$. Hence the topology of $X$ is the weak topology induced by $\mathfrak{C}(X, F)$.

$(3) \Rightarrow(4)$. The hypothesis equivalently states that $\mathfrak{C}(X, F)$ separates points and closed subsets of $X$ and Lemma 2.4 implies that $\mathfrak{C}(X, F)$ separates points and closed subsets of $X$ if and only if $2 \mathfrak{B}(X, F)$ separates points and closed subsets of $X$.

$(4) \Rightarrow(1)$. This is just a translation of Definition 2.9(2).

If (5) holds, then surely (4) holds. If $F=\mathbb{R}$, from Theorem 2.5, (4) surely implies (5); the nontrivial part of the proof of (4) implying (5) when $F \neq \mathbb{R}$ is deferred until we have proved Theorem 2.11 . 
Surprisingly, if $F \neq \mathbb{R}$, there are simpler ways to recognize the property of complete $F$-regularity, and we will provide a couple of them in Theorems 2.11 and 2.14.

THEOREM 2.11. For $F \neq \mathbb{R}$, a topological space $X$ is completely $F$-regular if and only if it is zero dimensional.

Proof. If $X$ is completely $F$-regular, then from Theorem 2.10(3) $X$ has the weak topology induced by $\mathfrak{C}(X, F)$, and from Theorem 2.2(2) it follows that $X$ has a base for its topology of clopen sets, and hence is zero dimensional. Conversely, if $X$ is zero dimensional, then for any closed subset $A \subseteq X$ and any point $x \in X \backslash A$ there exists a clopen set $W$ such that $x \notin W \supseteq A$. However, since $W$ is clopen, the characteristic map $X \stackrel{X_{W}}{\longrightarrow}\{0,1\} \subseteq F$ is continuous and is therefore a member of $\mathfrak{C}(X, F)$ separating $A$ and $x$. Hence $X$ is completely $F$-regular.

Note that the "if" part of the proof of Theorem 2.11 uses the fact that the space is zero dimensional to construct the separating function $\chi_{W}$ from $\mathfrak{C}^{*}(X, F)$. This produces the deferred proof.

Proof $((4) \Rightarrow(5)$ in Theorem 2.10 when $F \neq \mathbb{R})$. Since (4) holds, it follows from the proved equivalence in Theorem 2.10 that $X$ is completely $F$-regular; also, since $F \neq \mathbb{R}$, it follows from Theorem 2.11 that $X$ is zero dimensional, and the proof of Theorem 2.11 shows that $\mathfrak{C}^{*}(X, F)$ separates points and closed subsets of $X$.

COROLlaRY 2.12. F is completely F-regular.

Proof. If $F=\mathbb{R}$, the proposition is known to be true; if $F \neq \mathbb{R}$, then from Theorem 2.2(2) it follows that $F$ is zero dimensional, and then using Theorem 2.11 the statement is proved.

COROLLARY 2.13. The property of complete F-regularity is productive and hereditary.

Proof. For $F=\mathbb{R}$, this is a well-known result; for $F \neq \mathbb{R}$, this is a well-known property of zero-dimensional spaces.

THEOREM 2.14. A topological space $X$ is completely $F$-regular if and only if it is homeomorphic to a subspace of a product of $F$.

Proof. The "if" part of the proposition follows directly from Corollary 2.13. Conversely, let $X$ be completely $F$-regular and $P=F^{\mathfrak{C}(X, F)}$ equipped with the product topology, and let $X \stackrel{p}{\rightarrow} P$ be the evaluation map, that is, $p(x)=\{f(x)\}_{f \in \mathbb{C}(X, F)}$, for any $x \in X$.

Since $X$ is completely $F$-regular, it follows that $p$ is one-to-one; also being a map into a product space with continuous projections, $p$ is continuous. Let $x \in U \subseteq X$ where $U$ is an open subset of $X$. Then from the complete $F$-regularity of $X$ there exists a continuous map $X \stackrel{g}{\rightarrow}[0,1] \subseteq F$ such that $g(x)=1$ and $X \backslash U \subseteq \mathscr{L}_{X, F}(g)$ if and only if $x \in g^{\leftarrow}((1 / 2,2)) \subseteq U$. Hence, $p(x) \in p(X) \cap \pi_{g}{ }^{\leftarrow}((1 / 2,2)) \subseteq p(U)$, where $P \stackrel{\pi_{g}}{\longrightarrow} F$ is the $g$ th projection map. This shows that $p(U)$ is open in $p(X)$. Hence, $p$ is a topological embedding of $X$ to a product of $F$, so that $X$ is homeomorphic to a subspace of a product of $F$. 
At this point we can recollect that, given a topological space $E$, a topological space $X$ is said to be E-completely-regular if and only if $X$ is homeomorphic to a subspace of a product of the space $E$ (see [13] and cf. [36]). Thus the summary of Theorems 2.14 and 2.11 is that for a topological space $X$, the following three conditions are equivalent:

(1) $X$ is zero dimensional,

(2) $X$ is completely $F$-regular for any ordered field $F \neq \mathbb{R}$,

(3) $X$ is $F$-completely-regular, for any ordered field $F \neq \mathbb{R}$.

We will now show that for the purposes of our study of a topological space in terms of the function ring $\mathfrak{C}(X, F)$, it will be enough to restrict ourselves to the class of completely $F$-regular topological spaces only.

THEOREM 2.15. For any topological space $X$, there exists a completely $F$-regular topological space $Y$ such that $\mathfrak{C}(X, F)$ is isomorphic to $\mathfrak{C}(Y, F)$ as lattice-ordered commutative rings with unity.

Furthermore, the restriction of this isomorphism to the subring $2 \mathfrak{3}(X, F)$ or $\mathfrak{C}^{*}(X, F)$ define isomorphisms onto the corresponding subrings $\mathfrak{B}(Y, F)$ and $\mathfrak{C}^{*}(Y, F)$, respectively, of $\mathfrak{C}(Y, F)$.

Proof. For $x, y \in X$, let $x \equiv y$ if and only if, for all $f \in \mathbb{C}(X, F), f(x)=f(y)$. Clearly this is an equivalence relation on $X$ and partitions $X$. Let, for any $x \in X,[x]$ denote the $\equiv$-equivalence class of $X$, and let $Y$ be the set of all $\equiv$-equivalence classes of $X$. Let $X \stackrel{v}{\rightarrow} Y$ be the quotient map defined by $v(x)=[x]$.

For any $f \in \mathfrak{C}(X, F), x, x^{\prime} \in X$, if $[x]=\left[x^{\prime}\right]$, then $f(x)=f\left(x^{\prime}\right)$. Thus for $f \in \mathfrak{C}(X, F)$ one has a function $Y \stackrel{\hat{f}}{\rightarrow} F$ defined by $\hat{f}([x])=f(x)$. Hence $f=\hat{f}_{\circ} \nu$.

Taking $C^{\prime}=\{\hat{f}: f \in \mathfrak{C}(X, F)\}$, we endow $Y$ with the weak topology induced by $C^{\prime}$. Then $X \stackrel{v}{\rightarrow} Y$ is a continuous map, and if $Y \stackrel{g}{\rightarrow} F$ is any continuous map, then the equation $\widehat{(g \circ v)}=g$ implies that $C^{\prime}=\mathfrak{C}(Y, F)$. Hence, from Theorem 2.10(3) it follows that $Y$ is completely $F$-regular.

Finally, the map $\mathfrak{C}(X, F) \stackrel{\sigma}{\rightarrow} \mathfrak{C}(Y, F)$ defined by $\sigma(f)=\hat{f}$ is the required isomorphism of lattice-ordered commutative rings with unity.

From the definition of $\hat{f}$ it is clear that $\hat{f}$ is bounded or has a precompact range if and only if $f$ is bounded or has a precompact range, respectively. Hence the isomorphism $\sigma$ restricts to an isomorphism of $\mathfrak{2}(X, F)$ and $\mathfrak{2} \mathfrak{B}(Y, F)$ and an isomorphism of $\mathfrak{C}^{*}(X, F)$ and $\mathfrak{C}^{*}(Y, F)$, completing the proof.

REMARK 2.16. In the case $F=\mathbb{R}$, the last part of the statement was an obvious consequence of the fact that the positive cone of $\mathbb{R}$ consists precisely of perfect squares, see [15, Theorems 1.6, 1.7, Corollary 1.8, Theorem 1.9, pages 12-14]. However, this is not true in any arbitrary ordered field:

(1) for the field $\mathbb{Q}$ of the rationals, the positive cone contains many elements that are not perfect squares, or

(2) in Example 2.8, $\boldsymbol{x}>\mathbf{0}$ but is not a perfect square.

However, the proof of Theorem 2.15 does not require this specific property of the reals, as exhibited in the argument.

Let $\mathfrak{k}$ denote any of the three rings $\mathfrak{C}(X, F), \mathfrak{2}(X, F)$, or $\mathfrak{C}^{*}(X, F)$. An ideal $I$ of $\mathfrak{k}$ is said to be fixed if and only if $\bigcap_{f \in I} \mathscr{E}_{X, F}(f) \neq \varnothing$ and free otherwise. For the special case of 
$\mathfrak{k}=\mathfrak{C}(X, F)$, we have a very special kind of an ideal which will be of much importance in this paper.

DEFINITION 2.17. An ideal $I$ of $\mathfrak{C}(X, F)$ is said to be a $z_{F}$-ideal if and only if, for any $g \in \mathfrak{C}(X, F), \mathscr{E}_{X, F}(g) \in \mathscr{E}_{X, F}(I)$ implies that $g \in I$.

REMARK 2.18. One can view $\mathscr{L}_{X, F}$ as a function defined on the set $\mathfrak{C}(X, F)$ and with $3(X, F)$ as its range, taking $f \in \mathfrak{C}(X, F)$ to its set of zeros, namely, $\mathscr{L}_{X, F}(f)$. Thus, Definition 2.17 essentially says that an ideal $I$ of $\mathfrak{C}(X, F)$ is a $z_{F}$-ideal if and only if $\mathscr{E}_{X, F} \leftarrow\left(\mathscr{E}_{X, F}(I)\right)=I$.

Since $3(X, F)$ is a lattice of subsets of $X$, one can define filters and ultrafilters, and these are called $\zeta_{F}$-filters and $\zeta_{F}$-ultrafilters on $X$, respectively. We complete this section with a list of properties for different kinds of ideals.

THEOREM 2.19. (1) The class of $z_{F}$-ideals is closed under arbitrary meets.

(2) Every $z_{F}$-ideal is an intersection of prime ideals.

(3) For any ideal $I$ of $\mathfrak{C}(X, F), \mathscr{L}_{X, F}(I)$ is a $z_{F}$-filter on $X$, and for any $z_{F}$-filter $\mathscr{F}_{F}$ on $X, \mathscr{L}_{X, F}{ }^{-}(\mathscr{F})$ is a $\zeta_{F}$-ideal of $\mathfrak{C}(X, F)$. In particular, there is a one-to-one correspondence between the $z_{F}$-ideals of $\mathfrak{C}(X, F)$ and the $z_{F}$-filters on $X$.

(4) Every maximal ideal of $\mathfrak{C}(X, F)$ is a $z_{F}$-ideal of $\mathfrak{C}(X, F)$.

(5) The correspondence stated in (3) restricts to a correspondence between the maximal ideals of $\mathfrak{C}(X, F)$ and the $z_{F}$-ultrafilters on $X$.

(6) An ideal $M$ of $\mathfrak{C}(X, F)$ is maximal if and only if, for any $f \in \mathfrak{C}(X, F)$, if $\mathscr{L}_{X, F}(f) \cap$ $\mathscr{L}_{X, F}(g) \neq \varnothing$ for any $g \in M$, then $f \in M$.

(7) Let $\mathfrak{k} \in\left\{\mathfrak{C}(X, F), \mathfrak{B}(X, F), \mathfrak{C}^{*}(X, F)\right\}$. Then the fixed maximal ideals of $\mathfrak{k}$ are precisely given by $M_{x}=\{f \in \mathfrak{k}: f(x)=0\}, x \in X$.

Furthermore, if the space $X$ is completely F-regular, then the map $x \mapsto M_{x}$ is one-toone.

(8) For $f, g \in \mathfrak{C}(X, F)$, if $f$ belongs to every maximal ideal of $\mathbb{C}(X, F)$ to which $g$ belongs, then $\mathscr{E}_{X, F}(g) \subseteq \mathscr{E}_{X, F}(f)$.

(9) An ideal I of $\mathbb{C}(X, F)$ is a $z_{F}$-ideal if and only if, for any $f \in \mathfrak{C}(X, F)$, if $f$ belongs to every maximal ideal of $\mathfrak{C}(X, F)$ to which some member of I belongs, then $f \in I$.

(10) Let $F$ and $G$ be ordered fields, let $\mathfrak{C}(X, F) \stackrel{\sigma}{\rightarrow} \mathfrak{C}(Y, G)$ be an isomorphism of commutative lattice-ordered rings with unity, and let I be $a_{z_{F}}$-ideal of $\mathfrak{C}(X, F)$. Then $\sigma(I)$ is a ${ }_{G}$-ideal of $\mathfrak{C}(Y, G)$.

(11) The following are equivalent for a $z_{F}$-ideal I of $\mathfrak{C}(X, F)$ :

(a) I is a prime ideal;

(b) I contains a prime ideal;

(c) for all $f \in \mathfrak{C}(X, F)$ and $g \in \mathfrak{C}(X, F), f g=0$ implies $f \in I$ or $g \in I$,

(d) for any $f \in \mathfrak{C}(X, F)$, there exists a $Z \in \mathscr{L}_{X, F}(I)$ such that $f$ does not change its sign on $Z$.

(12) If $X$ is a compact topological space, then $\mathfrak{C}(X, F)$ cannot have any free ideals.

The converse is true under the additional hypothesis of complete F-regularity.

Proof. (1) Follows immediately from the definition.

(2) In any commutative ring $R$ with unity, given an ideal $I$, the intersection of all prime ideals that contain $I$ is $\left\{a \in R:(\exists n \in \mathbb{N})\left(a^{n} \in I\right)\right\}$; see [15, Theorem 0.18, page 7]. 
Applying this fact to the case of $\mathfrak{C}(X, F)$ and using the observation that for any $f \in$ $\mathfrak{C}(X, F)$ and $n \in \mathbb{N}, \mathscr{L}_{X, F}(f)=\mathscr{L}_{X, F}\left(f^{n}\right)$, one concludes that a $\mathfrak{Z}_{F}$-ideal in $\mathfrak{C}(X, F)$ is an intersection of prime ideals containing it.

(3) Since $\mathscr{L}_{X, F}\left(f^{2}+g^{2}\right)=\mathscr{L}_{X, F}(f) \cap \mathscr{L}_{X, F}(g)$ and $\mathscr{L}_{X, F}(f g)=\mathscr{L}_{X, F}(f) \cup \mathscr{L}_{X, F}(g)$, it follows that the image of any ideal $I$ under the map $\mathscr{L}_{X, F}$ is a $\zeta_{F}$-filter. The converse that given any $\zeta_{F}$-filter the inverse image is a $\zeta_{F}$-ideal follows from the observation made in Remark 2.18. The same observation helps in concluding the one-to-one correspondence between the $\zeta_{F}$-ideals and the $z_{F}$-filters.

(4) Let $M$ be a maximal ideal and $f \in \mathfrak{C}(X, F)$. Then $\mathscr{L}_{X, F}(f) \in \mathscr{L}_{X, F}(M)$ if and only if $f \in \mathscr{L}_{X, F}{ }^{\leftarrow}\left(\mathscr{L}_{X, F}(M)\right)$. Thus $\mathscr{L}_{X, F}{ }^{\leftarrow}\left(\mathscr{L}_{X, F}(M)\right)$ is a $\breve{Z}_{F}$-ideal containing $M$, from (3). Therefore, from the maximality of $M, M=\mathscr{L}_{X, F}{ }^{-}\left(\mathscr{L}_{X, F}(M)\right)$. Hence $M$ is a $\mathfrak{Z}_{F}$-ideal.

(5) Immediate from (3) and (4).

(6) Let $M$ be a maximal ideal and $f \in \mathfrak{C}(X, F)$ such that for any $g \in M$, $\mathscr{L}_{X, F}(f) \cap$ $\mathscr{E}_{X, F}(g) \neq \varnothing$. Then $\mathscr{E}_{X, F}(M) \cup\left\{\mathscr{E}_{X, F}(f)\right\}$ is a $\mathfrak{\zeta}_{F}$-filter-base on $X$. Hence from $(5), \mathscr{L}_{X, F}(f)$ $\in \mathscr{E}_{X, F}(M)$. Therefore from (4), $f \in M$.

Conversely, if $N$ is another ideal of $\mathfrak{C}(X, F)$ containing $M$, then for any $f \in N, \mathscr{E}_{X, F}(f) \cap$ $\mathscr{L}_{X, F}(g) \neq \varnothing$, for any $g \in M$. Hence from the hypothesis, $f \in M$ implying $N=M$. This proves the maximality of $M$.

(7) Choose and fix any $x \in X$ and consider the function $\mathfrak{k} \underset{\sigma_{x}}{\longrightarrow} F$ defined by $\sigma_{x}(f)=$ $f(x)$. Clearly this is an onto ring homomorphism with $\operatorname{ker}\left(\sigma_{x}\right)=\{f \in \mathfrak{k}: f(x)=0\}=$ $M_{x}$. Since $F$ is a field, it follows that $M_{x}$ is a maximal ideal of $k$, and indeed a fixed maximal ideal of $\mathfrak{k}$.

If $N$ is any fixed ideal of $k$, then there exists an $x \in X$ such that $x \in \bigcap_{f \in N} \mathscr{E}_{X, F}(f)$, and thus $N \subseteq M_{x}$. Thus, if $N$ is a fixed maximal ideal, then $N=M_{x}$, for some $x \in X$.

If $X$ is completely $F$-regular and $x, y \in X$ are two distinct points of $X$, then there exists an $f \in \mathfrak{k}$ such that $f(x)=0 \neq 1=f(y)$, and thus $f \in M_{x} \backslash M_{y}$, showing $M_{x} \neq M_{y}$. Thus, in the case when $X$ is completely $F$-regular, the map $x \mapsto M_{X}$ is one-to-one.

(8) If $f, g \in \mathfrak{C}(X, F)$ such that for any maximal ideal $M$ of $\mathfrak{C}(X, F), f \in M$ whenever $g \in M$, then for any $x \in \mathscr{L}_{X, F}(g)$, one has $g \in M_{X}$, which is a maximal ideal from (7). Hence, from our assumption, $f \in M_{X}$ if and only if $f(x)=0$ and $x \in \mathscr{L}_{X, F}(f)$, proving $\mathscr{L}_{X, F}(g) \subseteq \mathscr{E}_{X, F}(f)$.

(9) Let $I$ be a $z_{F}$-ideal and let $f \in \mathfrak{C}(X, F)$ be such that for any maximal ideal $M, f \in M$ whenever there exists a $g \in I$ such that $g \in M$. Hence, $\mathscr{L}_{X, F}(g) \subseteq \mathscr{L}_{X, F}(f)$, from (8), and hence $\mathscr{L}_{X, F}(f) \in \mathscr{L}_{X, F}(I)$, from (3). Therefore $f \in I$.

Conversely, if $f \in \mathfrak{C}(X, F)$ such that $\mathscr{L}_{X, F}(f) \in \mathscr{E}_{X, F}(I)$, then there exists a $g \in I$ such that $\mathscr{L}_{X, F}(f)=\mathscr{L}_{X, F}(g)$, and thus for any maximal ideal $M$ of $\mathfrak{C}(X, F), f \in M$ whenever $g \in M$. Hence from the hypothesis, $f \in I$. Hence $I$ is a $z_{F}$-ideal.

(10) Follows from (9) and the fact that maximal ideals of a ring are algebraic invariants.

(11) The implications $(\mathrm{a}) \Rightarrow(\mathrm{b}) \Rightarrow(\mathrm{c})$ are immediate, and we only prove $(\mathrm{c}) \Rightarrow(\mathrm{d}) \Rightarrow(\mathrm{a})$.

(c) $\Rightarrow(\mathrm{d})$. For any $f \in \mathfrak{C}(X, F),(f \wedge \mathbf{0})(f \vee \mathbf{0})=\mathbf{0}$, implying either $f \wedge \mathbf{0} \in I$ or $f \vee \mathbf{0} \in I$. Hence (d) follows.

(d) $\Rightarrow($ a). Let $f, g \in \mathfrak{C}(X, F)$ such that $f g \in I$. Let $h=|f|-|g|$. By the hypothesis, there exists a $Z \in \mathscr{L}_{X, F}(I)$ such that $h$ does not change sign on $Z$. Let $h$ be nonnegative on $Z$. 
Then $Z \cap \mathscr{L}_{X, F}(f) \subseteq \mathscr{L}_{X, F}(g)$ implies $Z \cap \mathscr{L}_{X, F}(f g) \subseteq \mathscr{L}_{X, F}(g)$ which implies $\mathscr{L}_{X, F}(g) \in$ $\mathscr{L}_{X, F}(I)$, using (3). Hence $g \in I$.

(12) If $I$ is a free ideal of $\mathfrak{C}(X, F)$, then $\mathscr{E}_{X, F}(I)$ is a family of closed subsets of $X$ with the finite intersection property and having an empty intersection. Hence $X$ cannot be compact.

Conversely, let $X$ be completely $F$-regular and noncompact. Then, there exists a family $\mathscr{F}_{F}$ of closed subsets of $X$ with the finite intersection property and having an empty intersection. By complete $F$-regularity, from Theorem 2.10(2) it follows that we might as well consider $\mathscr{F}$ to be a family of zero sets in $X$ with respect to $F$. Then $\mathscr{F}$ is a $z_{F}$-filter-base for a free $z_{F}$-filter.

Note that it follows from Theorem 2.19(11) that any prime ideal $P$ of $\mathfrak{C}(X, F)$ can be extended to a unique maximal ideal, so that each of the rings $\mathfrak{C}(X, F) / P$ is a local ring.

3. Compactifications. Given a commutative ring $K$ with unity, let the set of all its maximal ideals be denoted by $2 \mathfrak{b}$, and let, for any $a \in K, 2 \mathfrak{W}_{a}=\{M \in 2 \mathfrak{b}: a \in M\}$. It is easy to see that $\left\{2 \mathfrak{W}_{a}: a \in K\right\}$ yields a base for the closed subsets of some unique topology on 2b, often referred to as the Stone topology or as the hull-kernel topology. $2 \mathrm{~b}$ equipped with the hull-kernel topology is called the structure space of the commutative ring $K$. The following facts are true for the structure space.

(1) For any $x \subseteq 2 \mathfrak{b}, \mathrm{cl}_{2 \mathfrak{b}}(x)=\{M \in 2 \mathfrak{b}: M \supseteq \bigcap x\}$ (the name hull-kernel topology is derived from this property).

(2) For any $x \subseteq 2 \mathfrak{W}, x$ is dense in $2 \mathfrak{b}$ if and only if $\bigcap x=\bigcap 2 \mathfrak{b}$.

(3) $2 \mathfrak{b}$ is a compact $T_{1}$ space.

(4) $2 b$ is Hausdorff if and only if, for every pair of distinct maximal ideals $M$ and $N$ of $K$, there exist points $a, b \in K$ such that $a \notin M, b \notin N$, and $a b \in \bigcap 2 \mathfrak{b}$. Thus, the structure space of $\mathbb{Z}$ is not Hausdorff, while if $X$ is completely $F$-regular, then the structure space of $\mathfrak{C}(X, F)$ is Hausdorff.

For a much more detailed account of structure spaces, see [15, Example 7A, page 108, Examples $\mathrm{M}$ and N, page 111].

We will denote by $2 \mathfrak{W}_{X, F}, 2 \mathfrak{W}_{X, F}^{6}$, and $\mathfrak{W}_{X, F}^{*}$ the structure spaces of the rings $\mathfrak{C}(X, F)$, $\mathfrak{2}(X, F)$, and $\mathfrak{C}^{*}(X, F)$, respectively. Also, we will have the maps $X \stackrel{\mathfrak{p}_{X, F}}{\longrightarrow} 2 \mathfrak{n}_{X, F}, X \stackrel{\mathfrak{p}_{X, F}^{\mathfrak{G}}}{\longrightarrow} 2 \mathfrak{n}_{X, F}^{\mathfrak{G}}$, and $X \stackrel{\mathfrak{p}_{X, F}^{*}}{\longrightarrow} 2 \mathfrak{V}_{X, F}^{*}$ defined by similar rules, $x \mapsto M_{X}$. It is clear that if $X$ is completely $F$ regular, then each of these maps are embeddings of $X$ into a compact $T_{1}$ space.

THEOREM 3.1. If $X$ is a completely F-regular topological space, then $2 \mathfrak{W}_{X, F}$ is a Hausdorff compactification of $X$ with $X \stackrel{\mathfrak{p}_{X, F}}{\longrightarrow} 2 \mathfrak{W}_{X, F}$ as the embedding.

Furthermore, if $Y$ is any compact completely F-regular topological space and $X \stackrel{f}{\rightarrow} Y$ is a continuous map, then there exists a unique map $2 \mathfrak{n}_{X, F} \stackrel{f^{2 n_{X, F}} \longrightarrow}{\longrightarrow}$ such that $f=f^{2 \mathfrak{n}_{X, F}}$ 。 $\mathfrak{p}_{X, F}$.

Proof. The first part follows easily from the properties of a structure space and complete $F$-regularity of $X$.

For the latter, let for any maximal ideal $M$ of $\mathfrak{C}(X, F), \widehat{M}=\left\{g \in \mathfrak{C}(Y, F): g_{\circ} f \in M\right\}$. It is clear that $\widehat{M}$ is a prime ideal of $\mathfrak{C}(Y, F)$, and hence from Theorem 2.19(11) it follows that it is contained within a unique maximal ideal; also, as $Y$ is compact, it follows that 
each of the maximal ideals is fixed. Hence there exists a unique $y \in Y$ such that $\widehat{M} \subseteq M_{y}$. Define $f^{2 n_{X, F}}(M)=y$. The continuity of $f^{2 n_{X, F}}$ and the proof of the factorization of $f$ through $\mathfrak{p}_{X, F}$ follow from a routine verification.

Thus, $2 \mathfrak{n}_{X, F}$ enjoys a similar extension property as satisfied by the Stone-Čech compactification $\beta X$ of $X$. We will come back to this property after we have settled some other small issues related to structure spaces.

REMARK 3.2. (1) In the classical case when $F=\mathbb{R}$, one immediately concludes that $2 \mathfrak{V}_{X, \mathbb{R}}$ is completely $\mathbb{R}$-regular, that is, Tychonoff, because any compact Hausdorff space is known to be normal and hence Tychonoff. In the case when $F \neq \mathbb{R}, 2 n_{X, F}^{*}$ will be shown to be completely $F$-regular; see Theorem 3.12 . The complete $F$-regularity of $2 \mathfrak{n}_{X, F}$ or $2 \mathrm{~V}_{X, F}^{6}$ is yet to be decided and remains open.

(2) Since the rings $\mathfrak{C}(X, F), \mathfrak{B}(X, F)$, and $\mathfrak{C}^{*}(X, F)$ are examples of $f$-rings with bounded inversion, it follows from [4, Proposition 3.3 and Remark 3.5] that the structure spaces of these rings are Hausdorff (the last author is thankful to Professor Banaschewski for showing him during a conversation that the maximal $l$-ideals of an $f$-ring with bounded inversion are precisely the maximal ideals, and/or drawing his attention to one of his papers where this spectrum has been investigated).

From Theorem 2.15 it follows that there exists a compact completely $F$-regular topological space $Y$ such that the rings $\mathfrak{C}\left(2 \mathfrak{W}_{X, F}, F\right)$ and $\mathfrak{C}(Y, F)$ are isomorphic as latticeordered commutative rings with unity. Indeed the following theorem tells us that there cannot exist more than one such space, up to homeomorphism.

THEOREM 3.3. If

(1) $F, G$ are ordered fields,

(2) $X$ is a compact completely $F$-regular topological space,

(3) $Y$ is a compact completely $G$-regular topological space,

(4) the rings $\mathfrak{C}(X, F)$ and $\mathfrak{C}(Y, F)$ are isomorphic as lattice-ordered commutative rings with unity,

then the spaces $X$ and $Y$ are homeomorphic.

Proof. Since isomorphic rings have homeomorphic structure spaces, it follows that the spaces $\mathfrak{w}_{X, F}$ and $\mathfrak{w}_{Y, G}$ are homeomorphic. Since $X$ is compact and completely $F$ regular, $X$ and $2 \mathfrak{n}_{X, F}$ are homeomorphic; similarly, the spaces $Y$ and $2 \mathfrak{n}_{Y, G}$ are homeomorphic. Hence $X$ and $Y$ are homeomorphic.

REMARK 3.4. This is a two-fold generalization of the classical Banach-Stone theorem: on the one hand, it generalizes from the special case of real-valued continuous functions and, on the other hand, the assumed hypothesis does not depend on the range field.

One can also obtain the familiar Gelfand-Kolmogoroff theorem in much the same way as in the classical case as illustrated in the following.

THEOREM 3.5. For any completely $F$-regular topological space $X$, the maximal ideals of $\mathfrak{C}(X, F)$ are precisely $M^{p}$, where

$$
M^{p}=\left\{f \in \mathfrak{C}(X, F): p \in \operatorname{cl}_{\mathfrak{W}_{X, F}}\left(\mathfrak{p}_{X, F}\left(\mathscr{L}_{X, F}(f)\right)\right)\right\}, \quad p \in \mathfrak{W}_{X, F} .
$$

Going back to the extension property, we give the following definition. 
DEFINITION 3.6. A Hausdorff compactification $\alpha X$ is said to satisfy the $F$-extension property if and only if, for any compact completely $F$-regular topological space $Y$ and any continuous map $X \stackrel{f}{\rightarrow} Y$, there exists a map $\alpha X \stackrel{f^{\alpha}}{\rightarrow} Y$ such that $f=f^{\alpha}{ }_{\circ} \alpha$.

Since $X$ is densely embedded in $\alpha X$, it follows that the map $f^{\alpha}$ is unique. Let $\mathfrak{K}(X, F)$ denote the class of all those Hausdorff compactifications of $X$ which satisfy the $F$ extension property. Also, the uniqueness of the extension forces an isomorphism $\mathfrak{C}^{*}(X$, $F) \stackrel{t_{\alpha}}{\longrightarrow} \mathfrak{C}(\alpha X, F)$ of lattice-ordered commutative rings with unity, defined by $t_{\alpha}: f \mapsto f^{\alpha}$. This provides an alternative proof of the Hausdorffness of $2 \mathfrak{W}_{X, F}^{*}$.

The set $\mathfrak{k}(X, F)$ contains $2 \mathfrak{W}_{X, F}$ and $\beta X$. Also, if $\gamma X \geq \alpha X \in \mathfrak{k}(X, F)$, then there exists a $\gamma X \stackrel{h}{\rightarrow} \alpha X$ such that $\alpha=h_{\circ} \gamma$. Further, if $Y$ is a compact completely $F$-regular topological space and $X \stackrel{f}{\rightarrow} Y$ is continuous, then, from the extension property enjoyed by $\alpha X$, one has $f=f^{\alpha}{ }_{\circ} \alpha$. Hence $f=f^{\alpha}{ }_{\circ} h_{\circ} \gamma$, implying thereby that $\gamma X$ also enjoys a similar extension property and $f^{\gamma}=f^{\alpha}{ }_{0} h$. Thus we have proved the following.

THEOREM 3.7. $\mathfrak{K}(X, F)$ is a complete upper sub-semilattice of the complete join semilattice $\mathfrak{K}(X)$ of all Hausdorff compactifications of $X$.

We will now provide methods to construct completely $F$-regular members of $\mathfrak{k}(X)$, where $X$ is a completely $F$-regular topological space. For any $B \subseteq \mathbb{C}^{*}(X, F)$ which separates points and closed subsets of $X$, let $X \stackrel{e_{B}}{\longrightarrow} \prod_{f \in B} \mathrm{cl}_{F}(f(X))$ be the evaluation map, that is, $e_{B}(x)=\{f(x)\}_{f \in B}$, and let $e_{B} X$ be the closure of $e_{B}(X)$ in the space $\prod_{f \in B} \mathrm{cl}_{F}(f(X))$. Clearly then $e_{B} X$ is a completely $F$-regular member of $\mathfrak{k}(X)$. Indeed, if $\mathfrak{E}_{0}(X)$ is the collection of all completely $F$-regular members of $\mathfrak{k}(X)$, then we have the following.

THEOREM 3.8. $\alpha X \in \mathfrak{k}_{0}(X)$ if and only if there exists $a B \subseteq \mathfrak{C}^{*}(X, F)$ separating points and closed subsets of $X$ such that $\alpha X$ and $e_{B} X$ are equivalent as compactifications.

Proof. It is clear from the definition that if $B \subseteq \mathfrak{C}^{*}(X, F)$ such that $B$ separates points and closed subsets of $X$, then $e_{B} X$ is a member of $\mathfrak{E}_{0}(X)$. For the other part of the proof we will require Lemma 3.9, which is an extension of a similar result in [10].

LEMMA 3.9. Let $X$ be a completely $F$-regular topological space, $B_{1}, B_{2} \subseteq \mathfrak{C}^{*}(X, F)$, both separating points and closed subsets of $X$, and $\alpha X, \gamma X \in \mathfrak{K}_{0}(X)$; also let $C_{\alpha}=\{f \in$ $\left.\mathfrak{C}(X, F):(\exists \alpha X \stackrel{\hat{f}}{\rightarrow} F)\left(f=\hat{f}_{\circ} \alpha\right)\right\}$. Then the following statements are true.

(1) $B_{1} \subseteq B_{2}$ implies $e_{B_{1}} X \leq e_{B_{2}} X$.

(2) $B_{1} \subseteq C_{e_{B_{1}}}$.

(3) $\alpha X \leq \gamma X$ implies $C_{\alpha} \subseteq C_{\gamma}$.

(4) $\alpha X$ and $e_{C_{\alpha}} X$ are equivalent compactifications.

Proof. It is clear that $C_{\alpha} \subseteq \mathfrak{C}^{*}(X, F)$ and it separates points and closed subsets of $X$. The proofs of (1) and (3) are simple computations.

For (2), let $e_{B} X \stackrel{\pi_{f} e_{B} X}{\longrightarrow} \mathrm{cl}_{F}(f(X))$ be the restriction of the projection map to $e_{B} X$. Then for any $f \in B$ it is clear that $f=\pi_{f} 1_{e_{B} X_{\circ}} e_{B}$. 
For (4), it is enough to prove that $e_{C_{\alpha}} X \leq \alpha X$. Since, for any $f \in C_{\alpha}, \hat{f}_{\circ} \alpha=f=$ $\left(\pi_{f} 1_{e_{C_{\alpha}} X}\right)_{\circ} e_{C_{\alpha}}$, where $\pi_{f} 1_{e_{C_{\alpha}} X}$ is the restriction of the projection map to $e_{C_{\alpha}} X$, it follows that there exists a unique map $\alpha X \stackrel{\tilde{f}}{\rightarrow} \prod_{f \in C_{\alpha}} \mathrm{cl}_{F}(f(X))$ such that for each projection $\pi_{f}, \pi_{f}, \tilde{f}=\hat{f}$. Clearly then $\tilde{f}(\alpha X) \subseteq e_{C_{\alpha}} X$, so that the required inequality follows.

The proof of the theorem immediately follows from Lemma 3.9(4). The proof of Lemma 3.9 suggests an intimate connection between the poset of the subrings of $\mathfrak{C}^{*}(X, F)$ that separate points and closed subsets of $X$ and the poset of all completely $F$-regular members of $\mathfrak{K}(X)$, namely, $\mathfrak{K}_{0}(X)$; and the map $\alpha X \mapsto C_{\alpha}$ turns out to be an isomorphism. Hence, if $F \neq \mathbb{R}, \mathfrak{K}_{0}(X)$ is precisely the class of all zero-dimensional Hausdorff compactifications of $X,\left(\mathfrak{K}_{0}(X)\right.$ is a complete upper sub-semilattice of $\mathfrak{k}(X)$, and the greatest member of $\mathfrak{k}_{0}(X)$ is $\beta_{0} X$, the Banaschewski compactification of $X$. It is defined up to homeomorphism by an extension property similar to $\beta X$ : every continuous function on $X$ to a compact zero-dimensional Hausdorff topological space has a unique extension to $\beta_{0} X$.) So that we must have the following.

THEOREM 3.10. If $F \neq \mathbb{R}$, the compactifications $e_{\mathfrak{C}^{*}(X, F)} X$ and $\beta_{0} X$ are equivalent compactifications.

It is clear that $\beta_{0} X$ also has the $F$-extension property, if $F \neq \mathbb{R}$, so that we also have $\beta_{0} X \in \mathfrak{K}(X, F)$. But then, as any two members of $\mathfrak{k}(X, F)$ have isomorphic function rings, it follows from Theorem 3.3 that there cannot be more than one member in $\mathfrak{E}_{0}(X) \cap \mathfrak{K}(X, F)$. Furthermore, from the definition of the $F$-extension property it follows that for any $\alpha X \in \mathfrak{k}(X, F), \beta_{0}=\beta_{0}^{\alpha}$ 。 $\alpha$, implying $\beta_{0} X \leq \alpha X$. Hence we have the following.

THEOREM 3.11. Let $F \neq \mathbb{R}$. Then the following are true.

(1) $\mathfrak{K}_{0}(X) \cap \mathfrak{K}(X, F)=\left\{\beta_{0} X\right\}$.

(2) $\beta_{0} X$ is the smallest member of $\mathfrak{K}(X, F)$.

(3) $\mathfrak{K}(X, F)$ is a complete lattice in $\mathfrak{K}(X)$.

Thus, if $F \neq \mathbb{R}$, the rings $\mathfrak{C}^{*}(X, F)$ and $\mathfrak{C}\left(\beta_{0} X, F\right)$ are isomorphic as rings and hence $2 \mathfrak{W}_{X, F}^{*}$ is homeomorphic to $\beta_{0} X$, yielding the following.

THEOREM 3.12. For any $F \neq \mathbb{R}, \mathfrak{2}_{X, F}^{*}$ is homeomorphic to $\beta_{0} X$ and hence $2 \mathfrak{b}_{X, F}^{*}$ is completely F-regular.

REMARK 3.13. For $F=\mathbb{R}, 2 b_{X, F}^{*}$ is simply $\beta X$, and thus completely $F$-regular, so that, for any ordered field $F, 2 \mathfrak{W}_{X, F}^{*}$ is always completely $F$-regular.

4. Conclusion. We recall that a topological space $X$ is said to be strongly zero dimensional if and only if $X$ is a nonempty Tychonoff space and every open cover of $X$ by cozero sets in $X$ with respect to $\mathbb{R}$ has a finite open refinement of mutually disjoint sets (see [12, Chapter 6, page 360]). Clearly, the refinement is also a cover by cozero sets in $X$ with respect to $F$, for any ordered field $F$, as each of the sets in the refinement is a clopen set. The definition entails that every strongly zero-dimensional space 
TABLE 4.1. List of properties of the pair $(X, F)$.

List of Properties

\begin{tabular}{l|l}
\hline $\mathbb{P}$ & $\mathfrak{C}(X, F)=\mathfrak{b}(X, F)$ \\
\hline $\mathbb{P}$ & $\mathfrak{C}^{*}(X, F)$ and $\mathfrak{C}(X, F)$ determine the same family of zero sets in $X$ with respect to $F$ \\
\hline $\mathbb{P}$ & $2 \mathfrak{W}_{X, F}$ is completely $F$-regular \\
\hline $\mathbb{P}$ & $2 \mathfrak{V}_{X, F}^{\mathfrak{G}}$ is completely $F$-regular \\
\hline $\mathbb{P}$ & $\mathfrak{K}(X, F)$ has exactly two points \\
\hline $\mathbb{P}$ & Given one $\alpha X \in \mathfrak{K}(X), \alpha X \equiv 2 \mathfrak{W}_{X, F}$ as compactifications \\
\hline
\end{tabular}

is zero dimensional, but the converse need not be true (see [12, Section 6.2.20, page $365]$ ) and it is known that the Stone-Čech compactification of a Tychonoff space $X$ is zero dimensional if and only if $X$ is strongly zero dimensional (see [12, Theorem 6.2.12, page 362]).

Thus we have the following classification of a Tychonoff topological space $X$.

(1) $X$ is not zero dimensional.

(2) $X$ is strongly zero dimensional.

(3) $X$ is zero dimensional and not strongly zero dimensional, and for any ordered field $F$ other than $\mathbb{R}$, exactly one of the following conditions is satisfied:

(a) $\beta_{0} X=2 \mathfrak{W}_{X, F}<\beta X$;

(b) $\beta_{0} X<2 \mathfrak{W}_{X, F}<\beta X$;

(c) $\beta_{0} X<2 \mathfrak{n}_{X, F}=\beta X$.

Thus it follows that $\mathfrak{K}(X, F)$ is exactly a singleton if and only if either $F=\mathbb{R}$ or $X$ is strongly zero dimensional, and in all other cases it has at least two elements. No cardinal estimates for $\mathfrak{k}(X, F)$ could be ascertained, and none of the known examples of zerodimensional but not strongly zero-dimensional spaces (see [9, 21, 22, 23, 25, 26]) could be classified in the exhibited three classes.

Before we complete this paper, we propose some questions that seem to be new. The questions concern certain properties of the pair $(X, F)$, where $X$ is a topological space, preferably a completely $F$-regular topological space, and $F$ is an ordered field. For instance, one such property is " $\mathfrak{K}(X, F)$ is a singleton", and it was shown that this property holds if and only if either $F=\mathbb{R}$ or $X$ is strongly zero dimensional. The properties are listed in Table 4.1, and the questions for a property $\mathbb{P}$ are as follows.

QUESTION 4.1. Given a topological space $X$, is it possible to provide a construction of an ordered field $F$ such that $\mathbb{P}$ is satisfied?

QUESTION 4.2. Given an ordered field $F$, is it possible to provide a construction of a topological space $X$ such that $\mathbb{P}$ is fulfilled?

QUeSTION 4.3. Is it possible to characterize all the pairs $(X, F)$ such that $\mathbb{P}$ holds? 
Apart from these, another question remains unanswered.

QUESTION 4.4. To which of the classes of zero-dimensional but not strongly zerodimensional spaces discussed above do the spaces discussed in [9, 21, 22, 25] belong?

ACKNOWLEDGMENTS. The authors are thankful to Professor Hans-Peter Künzi, Department of Mathematics and Applied Mathematics, University of Cape Town, for his valuable suggestions at many stages during the preparation of this paper. The research of the last author was supported in the first part by the University Grants Commission, New Delhi, and in the final part by NRF Research Grant in the University of Cape Town. The last author further acknowledges his teacher Professor V. Saroja, without whose constant support the present work would not have been possible.

\section{REFERENCES}

[1] S. K. Acharyya, K. C. Chattopadhyay, and G. G. Ray, Hemirings, congruences and the StoneČech compactification, Simon Stevin 67 (1993), no. suppl., 21-35.

[2] - Hemirings, congruences and the Hewitt realcompactification, Bull. Belg. Math. Soc. Simon Stevin 2 (1995), no. 1, 47-58.

[3] G. Bachman, E. Beckenstein, L. Narici, and S. Warner, Rings of continuous functions with values in a topological field, Trans. Amer. Math. Soc. 204 (1975), 91-112.

[4] B. Banaschewski, Pointfree topology and the spectra of $f$-rings, Ordered Algebraic Structures (Curaçao, 1995), Kluwer Academic Publishers, Dordrecht, 1997, pp. 123-148.

[5] _ The Real Numbers in Pointfree Topology, Textos de Matemática. Série B, vol. 12, Departamento de Matemática, Universidade de Coimbra, Coimbra, 1997.

[6] _ Gelfand and exchange rings: their spectra in pointfree topology, Arab. J. Sci. Eng. Sect. C Theme Issues 25 (2000), no. 2, 3-22.

[7] _ f f -rings and the Stone-Weierstrass theorem, Order 18 (2001), no. 2, 105-117.

[8] G. Birkhoff, Lattice Theory, American Mathematical Society Colloquium Publications, vol. 25, American Mathematical Society, New York, 1948.

[9] S. Broverman, Another realcompact, 0-dimensional, non- $N$-compact space, Proc. Amer. Math. Soc. 69 (1978), no. 1, 156-158.

[10] R. E. Chandler, Hausdorff Compactifications, Lecture Notes in Pure and Applied Mathematics, vol. 23, Marcel Dekker, New York, 1976.

[11] G. De Marco and M. Richter, Rings of continuous functions with values in a non-archimedean ordered field, Rend. Sem. Mat. Univ. Padova 45 (1971), 327-336.

[12] R. Engelking, General Topology, Sigma Series in Pure Mathematics, vol. 6, Heldermann Verlag, Berlin, 1989.

[13] R. Engelking and S. Mrówka, On E-compact spaces, Bull. Acad. Polon. Sci. Sér. Sci. Math. Astronom. Phys. 6 (1958), 429-436.

[14] I. Gelfand and A. Kolmogoroff, On rings of continuous functions on topological spaces, Dokl. Akad. Nauk SSSR 22 (1939), 11-15.

[15] L. Gillman and M. Jerison, Rings of Continuous Functions, The University Series in Higher Mathematics, D. Van Nostrand Company, Princeton, 1960.

[16] M. Henriksen, On the equivalence of the ring, lattice, and semigroup of continuous functions, Proc. Amer. Math. Soc. 7 (1956), 959-960.

[17] _ A survey of $f$-rings and some of their generalizations, Ordered Algebraic Structures (Curaçao, 1995), Kluwer Academic Publishers, Dordrecht, 1997, pp. 1-26.

[18] E. Hewitt, Rings of real-valued continuous functions. I, Trans. Amer. Math. Soc. 64 (1948), 45-99.

[19] I. Kaplansky, Topological rings, Bull. Amer. Math. Soc. 54 (1948), 809-826. 
[20] A. N. Milgram, Multiplicative semigroups of continuous functions, Duke Math. J. 16 (1949), 377-383.

[21] S. Mrówka, Recent results on E-compact spaces, TOPO 72-General Topology and Its Applications, Lecture Notes in Math., vol. 378, Springer-Verlag, Berlin, 1974, pp. 298-301.

[22] A. Mysior, Two easy examples of zero-dimensional spaces, Proc. Amer. Math. Soc. 92 (1984), no. 4, 615-617.

[23] P. Nyikos, Prabir Roy's space $\Delta$ is not N-compact, General Topology and Appl. 3 (1973), 197-210.

[24] R. S. Pierce, Rings of integer-valued continuous functions, Trans. Amer. Math. Soc. 100 (1961), 371-394.

[25] P. Roy, Failure of equivalence of dimension concepts for metric spaces, Bull. Amer. Math. Soc. 68 (1962), 609-613.

[26] _ Nonequality of dimensions for metric spaces, Trans. Amer. Math. Soc. 134 (1968), 117-132.

[27] T. Shirota, A class of topological spaces, Osaka Math. J. 4 (1952), 23-40.

[28] _ A generalization of a theorem of I. Kaplansky, Osaka Math. J. 4 (1952), 121-132.

[29] M. H. Stone, Applications of the theory of Boolean rings to general topology, Trans. Amer. Math. Soc. 41 (1937), no. 3, 375-481.

[30] - A general theory of spectra. I, Proc. Natl. Acad. Sci. USA 26 (1940), 280-283.

[31] On the compactification of topological spaces, Ann. Soc. Polon. Math. 21 (1948), 153-160.

[32] V. I. Varankina and E. M. Vechtomov, On sheaves of algebras of functions generated by a mapping of topological spaces, Abelian Groups and Modules, No. 11, 12, Tomsk Gos. Univ., Tomsk, 1994, pp. 63-69.

[33] E. M. Vechtomov, An isomorphism of the multiplicative semigroups of algebras of continuous functions with compact support, Uspekhi Mat. Nauk 33 (1978), no. 5(203), 175-176.

[34] _ The module of all functions over a ring of continuous functions, Mat. Zametki 28 (1980), no. 4, 481-490.

[35] _ Projective and injective ideals of rings of continuous functions, Abelian Groups and Modules, Tomsk Gos. Univ., Tomsk, 1980, pp. 19-30.

[36] __ Definability of E-compact spaces in terms of partially ordered sets of ideals of rings of continuous functions, Abelian Groups and Modules, No. 7, Tomsk Gos. Univ., Tomsk, 1988, pp. 20-30.

[37] _ Rings of continuous functions with values in topological skew fields, Mat. Issled. (1988), no. 105, 45-52.

[38] _ Some properties of the ideals of rings of continuous functions, Topological Spaces and Their Mappings, Latv. Gos. Univ., Riga, 1989, pp. 40-49.

[39] _ Questions on the determination of topological spaces by algebraic systems of continuous functions, Algebra. Topology. Geometry, Itogi Nauki i Tekhniki, vol. 28, Akad. Nauk SSSR Vsesoyuz. Inst. Nauchn. i Tekhn. Inform., Moscow, 1990, pp. 3-46, translated in J. Soviet Math. 53 (1991), no. 2, 123-147.

[40] _ Rings of continuous functions. Algebraic aspects, Algebra. Topology. Geometry, Itogi Nauki i Tekhniki, vol. 29, Akad. Nauk SSSR Vsesoyuz. Inst. Nauchn. i Tekhn. Inform., Moscow, 1991, pp. 119-191, translated in J. Math. Sci. 71 (1994), no. 2, 2364-2408.

[41] On Gel'fand's and Kolmogorov's theorem on the maximal ideals of rings of continuous functions, Uspekhi Mat. Nauk 47 (1992), no. 5(287), 171-172.

[42] _ Rings of continuous functions and Gel'fand theory, Uspekhi Mat. Nauk 48 (1993), no. 1(289), 163-164, translated in Russian Math. Surveys 48 (1993), no. 1, 199-200.

[43] _ Rings of continuous functions and sheaves of rings, Uspekhi Mat. Nauk 48 (1993), no. 5(293), 167-168, translated in Russian Math. Surveys 48 (1993), no. 5, 187-188.

[44] - On the general theory of rings of continuous functions, Uspekhi Mat. Nauk 49 (1994), no. 3(297), 177-178, translated in Russian Math. Surveys 49 (1994), no. 3, 202-204. 
[45]__ Rings and sheaves, J. Math. Sci. (New York) 74 (1995), no. 1, 749-798.

[46] W. Więsław, Topological Fields, Marcel Dekker, New York, 1978.

S. K. Acharyya: Department of Pure Mathematics, University of Calcutta, 35 Ballygaunge Circular Road, Calcutta 700 019, West Bengal, India

E-mail addresses: sudipkumaracharyya@yahoo.co.in; skacharyya@rediffmai1.com

K. C. Chattopadhyay: Department of Mathematics, University of Burdwan, Burdwan 713 104, West Bengal, India

Partha Pratim Ghosh: School of Mathematical and Statistical Sciences, Howard College Campus, University of KwaZulu-Natal, Durban 4041, South Africa

Current address: Department of Mathematics and Applied Mathematics, University of Cape Town, Rondebosch 7701, Cape Town, South Africa

E-mail address: annapurnahazra@yahoo.com 


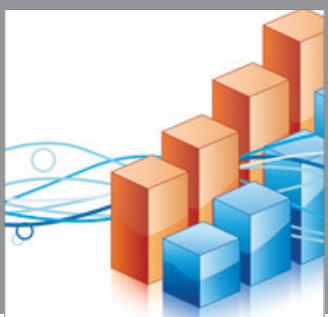

Advances in

Operations Research

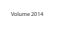

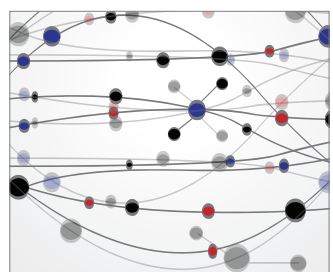

\section{The Scientific} World Journal
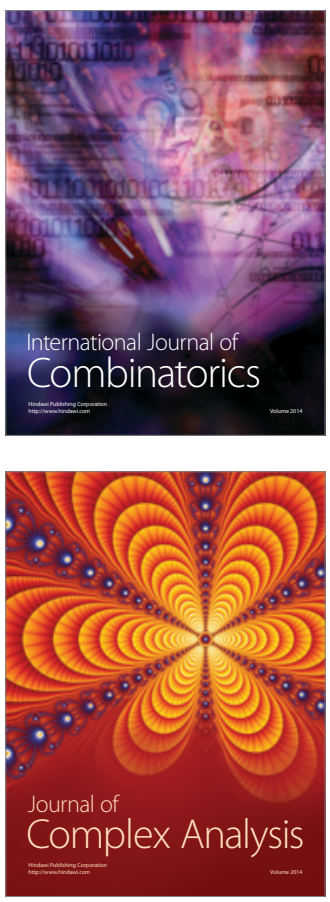

International Journal of

Mathematics and

Mathematical

Sciences
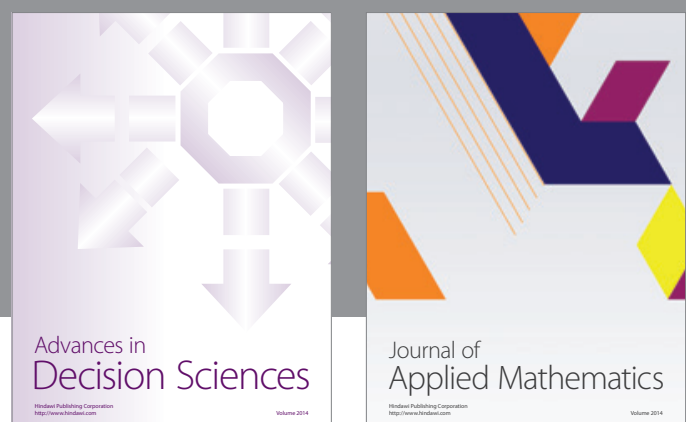

Journal of

Applied Mathematics
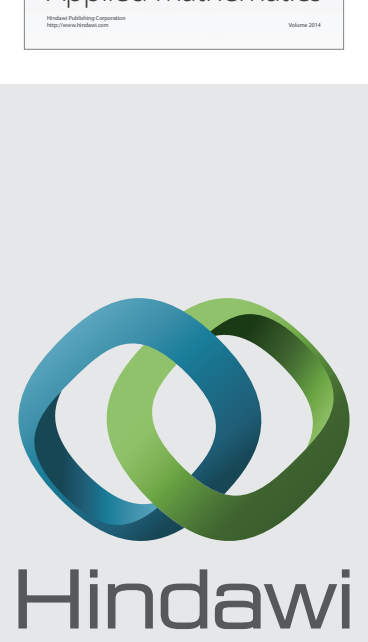

Submit your manuscripts at http://www.hindawi.com
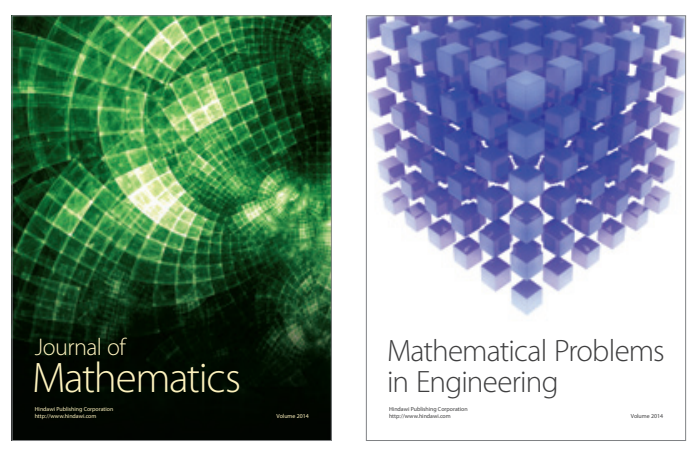

Mathematical Problems in Engineering
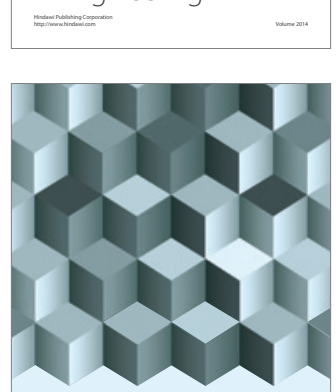

Journal of

Function Spaces
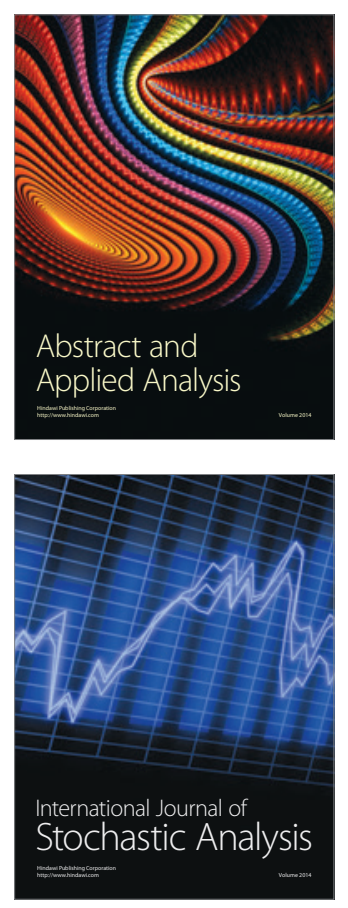

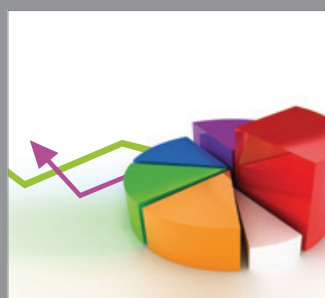

ournal of

Probability and Statistics

Promensencen
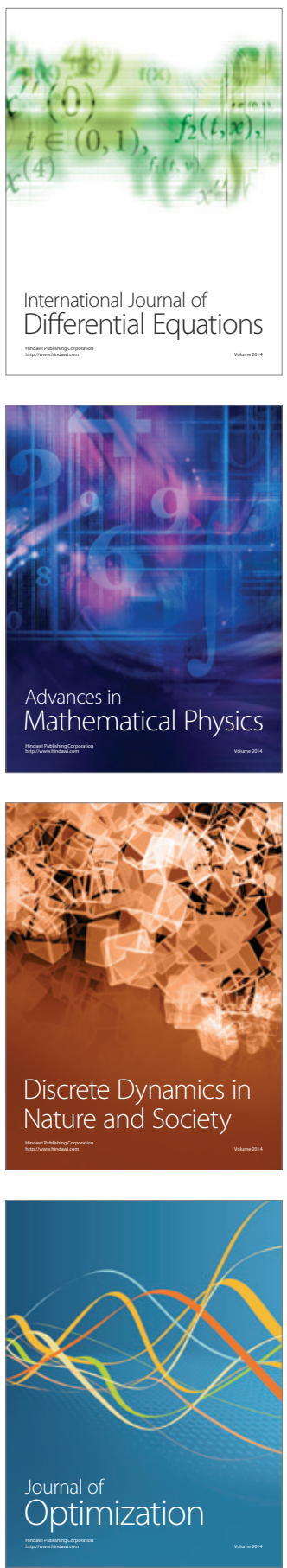